\title{
Pelo lanoso generalizado: reporte de un caso
}

\author{
MARÍA TRINIDAD HASBÚN Z. ${ }^{1,2}$, SERGIO GONZÁLEZ B. ${ }^{3}$ \\ 1. Servicio de Dermatología, Hospital Sótero del Río, Santiago, Chile. \\ 2. Servicio de Dermatología, Clínica Alemana de Santiago, Chile. \\ 3. Departamento de Anatomía-Patológica, Facultad de Medicina, Pontificia Universidad Católica de Chile. Santiago, Chile.
}

\begin{abstract}
Generalized woolly hair: case report

Introduction: Woolly hair (WH) is a rare abnormality of the hair shaft that can be localized or generalized and may be associated with cutaneous or extracutaneous abnormalities. Objective: To analyze a rare clinical case and emphasize the importance of physical examination. Case report: A three year old child with fine, light, short and curly hair is reported. Regarding family history, his mother reports alopecia since childhood and decreased growth rate of the hair of the scalp. The clinical picture is compatible with generalized hereditary woolly hair without associated anomalies. Conclusions: Woolly hair is a rare abnormality of the structure of the scalp hair. Electron microscopy allows the definitive diagnosis, however the use of dermoscopy is a practical and effective diagnostic tool in everyday practice.

(Key words: Woolly hair, scanning electron microscopy, cardiac abnormalities).

Rev Chil Pediatr 2013; 84 (6): 667-671
\end{abstract}

\section{RESUMEN}

Introducción: El pelo lanoso (PL) es una rara alteración del tallo piloso que puede ser localizada o generalizada y puede asociarse a alteraciones cutáneas o extracutáneas. Objetivo: Analizar un cuadro clínico de muy escasa frecuencia y enfatizar la importancia del examen físico en el enfrentamiento de ésta. Caso clínico: Preescolar de tres años de edad con pelo fino, claro, corto y rizado. En los antecedentes familiares, destacaba la madre con historia de alopecia desde la infancia y disminución de la velocidad de crecimiento del pelo del cuero cabelludo; cuadro compatible con pelo lanoso generalizado forma hereditaria, sin anomalías asociadas. Conclusiones: El pelo lanoso es una rara anormalidad del tallo piloso. El diagnóstico de certeza se obtiene mediante la microscopía electrónica, sin embargo, el uso de la dermatoscopía constituye una buena herramienta diagnóstica en la práctica diaria. Puede asociarse a anomalías cutáneas y extracutáneas, por lo cual el enfrentamiento clínico y estudio complementario es primordial para descartar anomalías asociadas.

(Palabras clave: Pelo lanoso, microscopía electrónica de barrido, anomalías cardíacas).

Rev Chil Pediatr 2013; 84 (6): 667-671

Recibido el 22 de diciembre de 2012, devuelto para corregir el 03 de junio del 2013, segunda versión 17 de septiembre de 2013, aceptado para publicación el 10 de octubre de 2013.

Este trabajo cumple con los requisitos sobre consentimiento /asentimiento informado, comité de ética, financiamiento, estudios animales y sobre la ausencia de conflictos de intereses según corresponda.

Correspondencia a:

Dra. Trinidad Hasbún Z.

mhasbun@alemana.cl 


\section{Introducción}

El término "pelo lanoso" se refiere a un tipo de anormalidad del tallo piloso caracterizada por un cabello fino, rizado y generalmente hipopigmentado. Fue reportada inicialmente en 1907 por Gossage ${ }^{1}$ y clásicamente se pueden subdividir en dos grandes grupos: la variante localizada o circunscrita (nevo de pelo lanoso) y la variante generalizada o difusa, dentro de la cual destaca el pelo lanoso hereditario autosómico dominante y pelo lanoso familiar autosómico recesivo ${ }^{2}$.

Las variantes hereditarias se pueden presentar en forma aislada o en asociación con otras alteraciones cutáneas o extracutáneas. De las manifestaciones cutáneas, las más frecuentes son queratosis pilar y queratodermia palmo-plantar y de las extra-cutáneas la asociación más importante es con alteraciones cardíacas, dentro de las cuales puede formar parte de distintos síndromes, como la enfermedad de Naxos (displasia arritmogénica ventricular derecha), Naxos-símil (displasia arritmogénica ventricular derecha asociado a desarrollo precoz de ampollas en rodillas, palmas y plantas) o el síndrome de Carvajal (cardiomiopatía dilatada) ${ }^{2}$.

El objetivo es presentar una patología de muy escasa frecuencia y enfatizar la importancia del examen físico en el enfrentamiento de ésta.

\section{Caso clínico}

Preescolar de sexo masculino, 3 años 2 meses de edad, con antecedentes de retraso leve del lenguaje, derivado de atención primaria por alteración en el crecimiento del pelo de cuero cabelludo. Al interrogatorio dirigido, madre refería disminución de la tasa de crecimiento del pelo desde el nacimiento, con un episodio de caída de pelo generalizada a los 7 meses de edad, después de lo cual comenzó a crecer pelo corto y rizado. En los antecedentes familiares, destacaba la madre con historia de alopecia desde la infancia y disminución de la velocidad de crecimiento del pelo del cuero cabelludo. El paciente era hijo único, sin an- tecedentes personales de problemas cardíacos ni antecedentes de otros familiares afectados.

Al examen físico, se observaba un niño de aspecto sano, con morfología facial conservada. Al examen dirigido de cuero cabelludo destacaba pelo fino, claro, corto y rizado. No se observaban folículos pilosos fracturados al examen físico. El pelo de las cejas también era escaso e hipopigmentado (figura 1).

No presentaba signos de queratosis pilar ni queratodermia palmo-plantar. Acudió con su madre, la cual presentaba alopecia de similares características, con pelo fino, corto, levemente rizado (figura 2 ).

La evaluación cardiológica y de laboratorio del paciente fueron normales. Se realizó

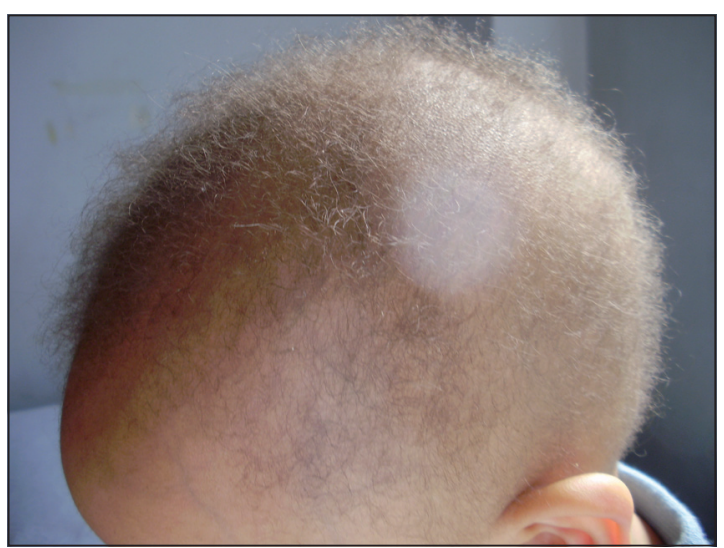

Figura 1. Pelo corto, rizado, generalizado.

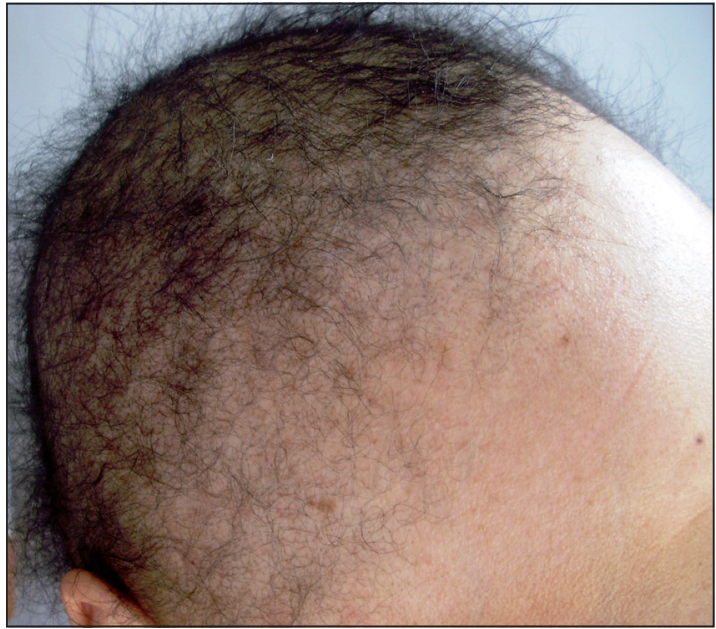

Figura 2. Pelo escaso y corto en la madre. 


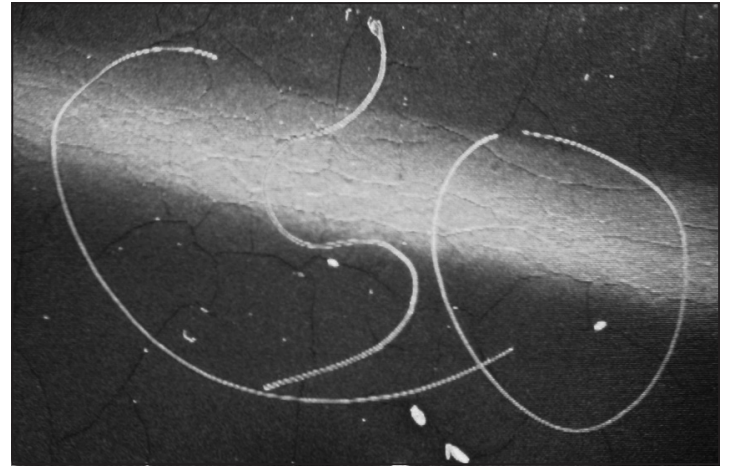

Figura 3. Microscopía electrónica: pelo delgado y curvo.

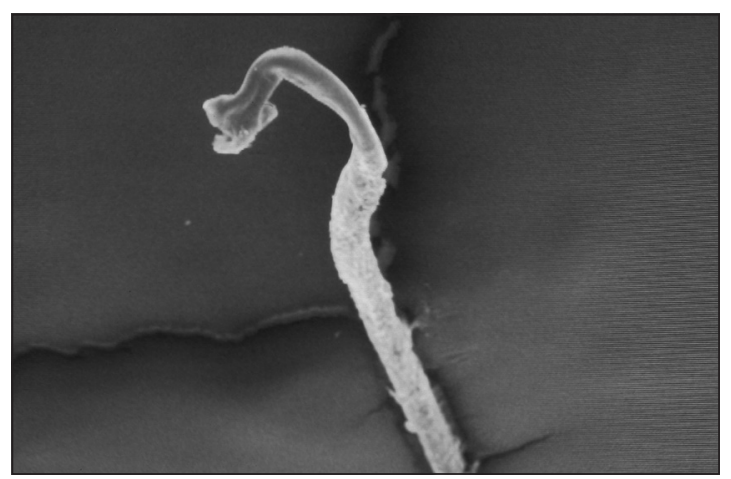

Figura 4. Microscopía electrónica: se aprecia pelo delgado y curvo con mayor acercamiento.

estudio de microscopía electrónica de barrido, que mostró pelos delgados y curvos, sin alteraciones de la cutícula ni presencia de cuerpos extraños compatible con pelo lanoso de tipo familiar (figuras 3 y 4 ).

\section{Discusión}

Las anormalidades del tallo piloso son un grupo de entidades que se caracterizan por alteraciones en la corteza interna y cutícula protectora del folículo piloso. Pueden ser congénitas o adquiridas y deben sospecharse en pacientes con textura o apariencia anormal del pelo o alteraciones en el crecimiento de éste, generalmente manifestado por la inhabilidad del pelo para alcanzar una longitud adecuada.

Un antecedente importante para la evaluación clínica es determinar si existe o no ruptura del folículo piloso (que refleja aumento de la fragilidad capilar). Esto se evidencia al examen físico por la presencia de folículos pilosos "fracturados". De esta manera, podemos encontrar alteraciones del tallo piloso con y sin fragilidad aumentada, los cuales a su vez pueden ser clasificados en congénitos o adquiridos ${ }^{1}$.

El término "pelo lanoso" alude a una entidad descrita por primera vez en 1907 por Gossage $^{2}$ y se caracteriza por un cabello fino, rizado e hipopigmentado. Posteriormente, en 1974, Hutchinson et $\mathrm{al}^{4}$, clasifica esta entidad en tres variantes: localizada o nevo de pelo lanoso, pelo lanoso hereditario autosómico dominante (PLAD) y pelo lanoso familiar autosómico recesivo (PLAR).

El nevo de pelo lanoso es fácilmente distinguible de las otras entidades por presentar un compromiso parcial y localizado en cuero cabelludo y un borde bien delimitado y circunscrito.

El pelo lanoso generalizado es aquel que compromete todo el cuero cabelludo y puede estar asociado, o no, a manifestaciones cutáneas y/o extracutáneas. Hutchinson et $\mathrm{al}^{4}$, describe a dos grupos familiares con PLAD, el cual afecta a ambos sexos y que exhibe cierta disminución del rizado en la adultez. Neild et $\mathrm{al}^{5}$, analiza la transmisión de PLAR y, a diferencia de la variante autosómica dominante, destaca como hallazgo constante la disminución de la velocidad de crecimiento del pelo y la hipopigmentación de éste.

Chien et $\mathrm{al}^{6}$, describe una familia con pelo lanoso hereditario y queratosis pilar, y propone un algoritmo de clasificación en base a la historia clínica y hallazgos al examen físico. Los dividen en dos grandes grupos: PL congénito con hallazgos asociados y PL congénito sin otros hallazgos asociados.

La patogénesis del PL aún no se encuentra del todo clara. Se postula que los desmosomas del folículo piloso (específicamente desmoplaquina, placoglobina y placofilina 1), proteínas celulares estructurales que mantienen adheridas a las células vecinas, se encontrarían alterados en esta patología, lo cual produciría una fragilidad en la unión celular y, por consiguiente, una disregulación en el desarrollo del folículo piloso ${ }^{7}$. 
La etiología exacta también es desconocida, sin embargo, existen alteraciones genéticas que han sido vinculadas al síndrome: en PLAR, genes LIPH (lipasa H), LPAR6 (receptor ácido lipofosfatídico 6) y gen de la desmogleína 4, y e PLAD, genes de la queratina $74,81,83$ y $86^{8}$.

Aquellos pacientes sin manifestaciones clínicas asociadas se pueden subdividir en los que presentan un compromiso circunscrito en el cuero cabelludo y que correspondería al nevo de pelo lanoso y aquellos con compromiso generalizado que, según las características clínicas y los antecedentes familiares, se clasifican en PLAD o PLAR ${ }^{6}$.

Por otra parte, están aquellos que presentan otras manifestaciones asociadas, dentro de las cuales uno de los hallazgos más importantes es la presencia o ausencia de queratosis pilar. En el caso que presente queratosis pilar e.n forma aislada, se puede interpretar como PL hereditario con QP asociada; sin embargo, si además del PL y la queratosis pilar presenta alteraciones cardíacas, se debe descartar el síndrome Cardio-fascio-cutáneo o, si presenta dismorfismo facial con estatura baja, cuello corto y alado, y manchas café con leche, el diagnóstico es compatible con Síndrome de Noonan ${ }^{6}$.

Otro hallazgo que puede estar asociado a PL es la presencia de queratodermia palmoplantar (QPP). La tríada de PL generalizado, QPP y cardiomiopatía ventricular derecha define al Síndrome de Naxos ${ }^{9}$, una patología autosómica recesiva rara, asociada a una mutación de la placoglobina ${ }^{10}$.

Por otra parte, la presencia PL y QPP con cardiomiopatía dilatada, compromiso de ventrículo izquierdo, debe hacernos sospechar el Síndrome de Carvajal, que se asocia a una mutación de la desmoplaquina ${ }^{11}$. Por lo tanto, en la práctica, la evaluación de los pacientes con PL generalizado debe ser exhaustiva: historia personal y familiar detallada; examen físico completo, identificando la presencia de queratosis pilar, queratodermia palmo-plantar, dismorfismo facial, estatura baja, alteraciones cardíacas, etc.; evaluación por cardiología, incluyendo ecocardiografía, sobre todo en aquellos pacientes que presenten manifestaciones asociadas, como queratodermia palmo-plan$\operatorname{tar}^{3}$.
Cabe destacar la dermatoscopía como herramienta de orientación diagnóstica en la práctica clínica. En el caso de PL se observa la imagen de ciclos alternantes de ondas cortas, a modo de una "serpiente arrastrándose" y la presencia de "pelos fracturados".

El examen de microscopía electrónica de barrido del pelo nos ayuda a confirmar el diagnóstico de pelo lanoso, pues se observa que el diámetro capilar es menor que el del cabello normal y en corte transversal es ovalado, no redondo ${ }^{12}$.

La tasa de crecimiento es normal, sin embargo, la fase de anágeno está acortada, por lo cual no crece más allá de algunos centímetros. Generalmente no existe fragilidad capilar asociada $^{12}$.

\section{Conclusión}

Mediante el abordaje metódico a través del análisis morfológico del folículo piloso, asociado a una evaluación clínica rigurosa y completa, podemos orientarnos a distintas alteraciones del tallo piloso, dentro de la cual el PL forma parte.

El presente caso clínico destaca la importancia de una historia clínica y examen físico exhaustivo, con énfasis en las manifestaciones tanto cutáneas como extracutáneas, para descartar otras anomalías asociadas y orientar la evaluación y mejor manejo de estos pacientes.

\section{Referencias}

1.- Mirmirani P, Huang K, Price V: A practical, algorithmic approach to diagnosing hair shaft disorders. Int J Dermatol 2011; 50: 1-12.

2.- Gossage AM: The inheritance of certain human abnormalities. Q J Med 1907; 1: 331-47.

3.- Soodabeh Z, Saeedeh F: A new cardiac manifestation associated with woolly hair: report of two cases of woolly hair, palmoplantar keratoderma, and mitral valve regurgitation. Int J Dermatol 2007; 46: 952-54.

4.- Hutchinson PE, Cairns RJ, Wells RS: Woolly hair: clinical and general aspects. Trans St Johns Hosp Dermatol Soc 1974; 60: 160-77.

5.- Neild VS, Pegum JS, Wells RS: The association of 
keratosis pilaris atrophicans and woolly hair, with and without Noonan's syndrome. Br J Dermatol 1984; 110: 357-62.

6.- Chien A, Valentine M, Sybert V: Hereditary woolly hair and keratosis pilaris. J Am Acad Dermatol 2006; 54 : S35-9.

7.- Cheng A, Bayliss $S$ : The genetics of hair shaft disorders. J Am Acad Dermatol 2008; 59: 1-22.

8.- Betz R, Cabral R, Christiano A, Sprecher E: Unveiling the Roots of Monogenic Genodermatoses: Genotrichoses as a Paradigm. Journal of Investigative Dermatology 2012; 132: 906-14.

9.- Rai R, Ramachandran V, Sundaram G, Rajendren $C$, Srinivas $R$ : Naxos disease: a rare occurrence of cardiomyopathy with woolly hair and palmoplantar keratoderma. Indian J Dermatol Venereol Leprol 2008; 74: 50-2.

10.- McKoy G, Protonotarios N, Crosby A, et al: Identification of a deletion in plakoglobin in arrhythmogenic right ventricular cardiomyopathy with palmoplantar keratoderma and woolly hair (Naxos disease). Lancet 2000; 17: 2119-24.

11.- Carvajal-Huerta L: Epidermolytic palmoplantar keratoderma with woolly hair and dilated cardiomyopathy. J Am Acad Dermatol 1998; 39: 418-21.

12.- Torres T, Machado S, Selores M: Woolly hair generalizado: caso clínico e revisão da literatura. An Bras Dermatol 2010; 85 (1): 97-100. 